\title{
Endovascular coil embolization of unruptured posterior communicating artery aneurysm
}

\author{
Mandy Binning, M.D., Zakaria Hakma, M.D., and Erol Veznedaroglu, M.D. \\ Department of Neurosurgery, Capital Institute for Neurosciences, Pennington, New Jersey
}

\begin{abstract}
The patient is a 60-year-old woman who presented to her primary care physician with new onset of headache. She was neurologically intact without cranial nerve deficit. An outpatient CT angiogram (CTA) revealed no subarachnoid hemorrhage, but showed a right-sided posterior communicating artery aneurysm measuring $11 \mathrm{~mm}$ by $10 \mathrm{~mm}$. Digitally subtracted cerebral angiography confirmed these measurements and showed that the aneurysm was amenable to endovascular coil embolization. The patient underwent aneurysm coiling without complication and was discharged to home on postoperative Day 1.

The video can be found here: http://youtu.be/MjOc3Zpv2K8.

(http://thejns.org/doi/abs/10.3171/2014.V2.FOCUS14179)
\end{abstract}

Key Words • endovascular • aneurysm • coiling • video

Manuscript submitted May 1, 2014.

Accepted June 4, 2014.

Please include this information when citing this paper: DOI: 10.3171/2014.V2.FOCUS14179.

Address correspondence to: Dr. Mandy J. Binning, Two Capital Way, Suite 456, Pennington, New Jersey, 08534. email: mandyjo binning@yahoo.com. 